\title{
Surfactant effects on the coalescence of a drop in a Hele-Shaw cell
}

\author{
Maxime Chinaud, Victor Voulgaropoulos, and Panagiota Angeli* \\ Department of Chemical Engineering, University College London, Torrington Place, London WC1E 7JE, United Kingdom
}

(Received 23 March 2016; published 1 September 2016)

\begin{abstract}
In this work the coalescence of an aqueous drop with a flat aqueous-organic interface was investigated in a thin gap Hele-Shaw cell. Different concentrations of a nonionic surfactant (Span 80) dissolved in the organic phase were studied. We present experimental results on the velocity field inside a coalescing droplet in the presence of surfactants. The evolution of the neck between the drop and the interface was studied with high-speed imaging. It was found that the time evolution of the neck at the initial stages of coalescence follows a linear trend, which suggests that the local surfactant concentration at the neck region for this stage of coalescence can be considered quasiconstant in time. This neck expansion can be described by the linear law developed for pure systems when the surfactant concentration at the neck is assumed higher than in the bulk solution. In addition, velocity and vorticity fields were computed inside the coalescing droplet and the bulk homophase using a high-speed shadowgraphy technique. The significant wall effects in the Hele-Shaw cell in the transverse axis cause the two vertical velocity components towards the singularity rupture point, from the drop and from the bulk homophase, to be of the same order of magnitude. This movement together with the neck expansion creates two pairs of counteracting vortices in the drop and in the bulk phase. The neck velocity is the average of the advection velocities of the two counteracting vortex pairs on each side of the neck. The presence of the surfactant slows down the dynamics of the coalescence, affects the propagation direction of the pair of vortices in the bulk phase, and reduces their size faster compared to the system without surfactant.
\end{abstract}

DOI: 10.1103/PhysRevE.94.033101

\section{INTRODUCTION}

When two bodies of the same liquid come into contact within a second immiscible liquid, a thin film of the surrounding immiscible phase is formed between them. This film will gradually drain until it finally ruptures and the coalescence of the two bodies is initiated. The film thinning and the coalescence rates depend on the fluid properties and the system conditions. Understanding the dynamics of these two phenomena is important for numerous multiphase applications that involve immiscible liquids and is thus still attracting the interest of many investigators [1-5]. The study of coalescence directly in the complex environment where drops appear (i.e., dispersions, emulsions, and separators) can prove challenging. However, investigating a controlled system of either an isolated drop coalescing with the flat surface of a bath of the same liquid (homophase), or with another drop, allows detailed observations to be made. This study will focus on coalescence between a droplet and a flat interface.

There are many investigations on the coalescence process following the rupture of the film. It has been shown that a liquid bridge is formed between two coalescing drops, which expands over the time. The dynamics of the bridge evolution and its validation against experimental data are still active research areas. Following the film rupture, two main regimes have been identified, which are characterized by different scaling laws, depending on whether the viscous or the inertial are the dominant forces that resist the surface tension forces driving

\footnotetext{
*p.angeli@ucl.ac.uk
}

Published by the American Physical Society under the terms of the Creative Commons Attribution 3.0 License. Further distribution of this work must maintain attribution to the author(s) and the published article's title, journal citation, and DOI. the coalescence at each step. In early efforts, Eggers et al. [6] developed a scaling law which states that when the neck of the bridge is sufficiently small, immediately after film rupture, viscous forces dominate. In this regime the capillary number can be taken equal to $\mathrm{Ca}=\mu V_{\text {visc }} / \gamma=1$ and the neck velocity is scaled as $V_{\text {visc }}=\gamma / \mu$, while the time scale is $t_{\text {visc }}=R \mu / \gamma$, where $V_{\text {visc }}$ and $t_{\text {visc }}$ are the velocity and corresponding time for the viscous regime, $R$ is the drop radius, $\mu$ is the viscosity of the coalescing drop, and $\gamma$ is the interfacial tension between the two fluids [6].

The crossover from the viscous to the inertial regime will happen at Reynolds numbers (based on the neck radius as the characteristic length and on the neck velocity of the viscous regime) close to 1 . In the inertial regime the Weber number, which now characterises the flow in the neck, is $\mathrm{We}=\left(\rho u_{\text {inert }}^{2} R / \gamma\right)^{1 / 2}=1$ and the neck velocity is scaled as $V_{\text {inert }}=(\gamma / \rho R)^{1 / 2}$, while the time scale is $t_{\text {inert }}=\left(\rho R^{3} / \gamma\right)^{1 / 2}$, where $\rho$ is the density of the coalescing liquid bodies [6]. As pointed out by Sprittles and Shikhmurzaev [7], the crossover will happen at neck radius $r=\mu^{2} / \rho \gamma$ (by substituting $V_{\text {inert }}$ for $\mathrm{Ca}=1$ or for $\mathrm{Re}=1$ ).

Several groups have worked on further developing this theory by providing experimental data to match the above efforts and better understand the liquid bridge hydrodynamics [7-10]. Notable is the work of Aarts et al. [11] who were able to experimentally capture pure viscous coalescence (first regime) by increasing the viscosity and decreasing the interfacial tension of the solutions used. Their results showed a linear expansion of the neck in the viscous regime which is more generally accepted for short time scales [12]. For the second regime, Aarts et al. [11] found coefficients in the range of 1.11-1.24 for different solutions, which are in agreement with some experimental data $[13,14]$ but deviate from the values of 1.62 found by Duchemin et al. [15] from numerical simulations and of 1.53 proposed by Chen et al. [16], who combined numerical and experimental data. 
As the surface tension is the driving force behind the coalescence mechanism, it is important to investigate how Marangoni stresses caused by surface tension gradients due to differences in temperature or composition of the fluids can affect it. In some works, surface active agents were used to induce this effect [17-19]. The scope of these studies was mainly to understand the diffusion of the surfactants and their effect on the interface deformation as well as the film drainage and coalescence times, while the information given on the coalescence dynamics after film rupture was limited. Blanchette et al. [20] investigated both experimentally and numerically the effect of surface tension gradients by varying the concentration of ethanol in aqueous mixtures, and linked the ratio of the surface tension between the two liquids to the evolution of the coalescence process. A subsequent numerical work [21] showed that the presence of surfactants limited the range where partial coalescence occurs. The accumulation of surfactants on the interface of a coalescing drop and the development of surface tension gradients which can affect the flow field during coalescence were shown.

Most of the coalescence studies available have been conducted in tanks or rectangular cells where the wall effects can be ignored. A few recent studies have focused on drop coalescence with an interface in a thin gap-quasi-two-dimensional cell (i.e., Hele-Shaw cell) [22,23]. Two-dimensional cells are used as approximations to creeping flows and flows in porous media, and are relevant to microfluidics applications. In this geometry, for steady-state and uniform flow, the velocity component along the depth of the cell can be neglected while the velocity component in the plane of measurement can be assumed to depend only on the $z$ dimension. Under these assumptions, the nonlinear term $(\overline{\overline{\vec{v}}} \bar{\nabla}) \vec{v}$ in the Navier-Stokes equations becomes zero and the flow can be considered two dimensional, for which the two velocity components along the $x$ and the $y$ axes can be averaged along the $z$ direction [24].

The linear scaling law for the viscous regime developed for three-dimensional (3D) systems was also found to describe the neck expansion in two-dimensional (2D) systems [12]. However, as discussed by Eri and Okumura [25], the dynamics of the bridge expansion for the inertial regime is different from the $3 \mathrm{D}$ case because the neck expansion is limited by the depth of the cell. For these cases, the surface energy per unit time has to be limited to a volume equal to $r D h$, where $h$ is the local radius of curvature of the neck and $D$ is the depth of the cell. By using the geometrical approximation $d \cong r^{2} / R$ a relationship was developed for the inertial regime, for $\sqrt{R D} \leqslant$ $R$, formulated as $r / \sqrt{R D}=\left(t / \tau_{f}\right)^{1 / 4}$ with $\tau_{f}=R \mu / \gamma$ [23].

The experimental studies of coalescence phenomena have mainly been based on high-speed visualizations. More elaborate approaches have been developed by Aarts et al. [11] who implemented laser scanning confocal microscopy with increased spatial resolution but low-time resolution $(50 \mathrm{~Hz})$ compared to high-speed imaging, while Fezzaa and Wang [26] studied the early stages of two coalescing water drops in air with an ultrafast $\mathrm{x}$-ray phase-contrast imaging system ( $2000 \mathrm{kHz}$ ). Case and Nagel [27] developed an innovative method to track coalescence dynamics based on the different impedance of the two liquids, which showed a new asymptotic regime that had not been predicted theoretically previously.
More recently, laser based advanced optical techniques, such as particle image velocimetry (PIV) and threedimensional PIV (tomo-PIV) have been used to acquire the velocity fields during drop coalescence. Experimental investigations of velocity fields during coalescence have mainly been conducted for the inertial regime after film rupture [28-31]. PIV approaches have been used for droplets of a typical size of $1 \mathrm{~cm}$, where measurements are carried out on a plane defined by a laser sheet, and the velocity fields are obtained by correlating two successive images. High-speed PIV approaches can reach sampling frequencies of the order of $1 \mathrm{kHz}$ [28]. The time difference between two consecutive images, however, is of the order of $0.1 \mathrm{~ms}$ which does not allow the large velocities occurring at the early stages of coalescence to be captured. For the early fast coalescence stages, classical PIV approaches using the straddling camera mode are more appropriate, where the time difference between two successive frames can be as short as $1 \mu \mathrm{s}$. The straddling mode systems, however, use cameras with frequencies of only a few $\mathrm{Hz}$ and cannot track the time evolution of the coalescence process. Such measurements imply that the PIV system needs to be triggered by the film rupture and the initiation of coalescence.

There have not been any experimental investigations of the velocity fields during coalescence in a Hele-Shaw cell. The typical velocity magnitudes encountered in the $2 \mathrm{D}$ cells are lower than in the 3D ones, which would allow high-speed PIV measurements after film rupture. In the present work, a similar but simpler experimental technique is implemented. High-magnification shadowgraphy measurements can be conducted with a constant LED backlight source for volumetric illumination. The measurement volume is then defined by the focal plane and the depth of field of the imaging system, which is smaller than the cell depth but of the same order of magnitude, while the time resolution is only limited by the acquisition frequency of the camera [32].

In this paper, the evolution of the velocity fields during coalescence in a Hele-Shaw cell is obtained for different surfactant concentrations. The effects of surfactants on the neck evolution and velocity fields are shown for coalescence in $2 \mathrm{D}$ cells. The experimental system together with the highspeed imaging and PIV measurement techniques are presented first, while the corresponding data analysis follows. The neck evolution dynamics for different surfactant concentrations are then discussed. Finally velocity, vorticity and shear rate fields and their evolution after the neck rupture are analysed for different surfactant concentrations.

\section{EXPERIMENTAL SETUP}

The coalescence experiments were carried out in a HeleShaw cell made from acrylic with dimensions $14 \times 8 \times$ $0.125 \mathrm{~cm}$, and corresponding aspect ratios of approximately $L / D=112$ and $W / D=64$ where $L, W$, and $D$ represent, respectively, the height, width, and depth of the cell (Fig. 1). Since $D \ll W<L$ the cell can be considered as a Hele-Shaw cell. In this particular geometry, the steady-state flow along the transverse direction (i.e., along the $z$ axis) can be considered as Poiseuille flow characterized by a parabolic velocity profile for Newtonian fluids [33]. The significant difference between the $z$ 


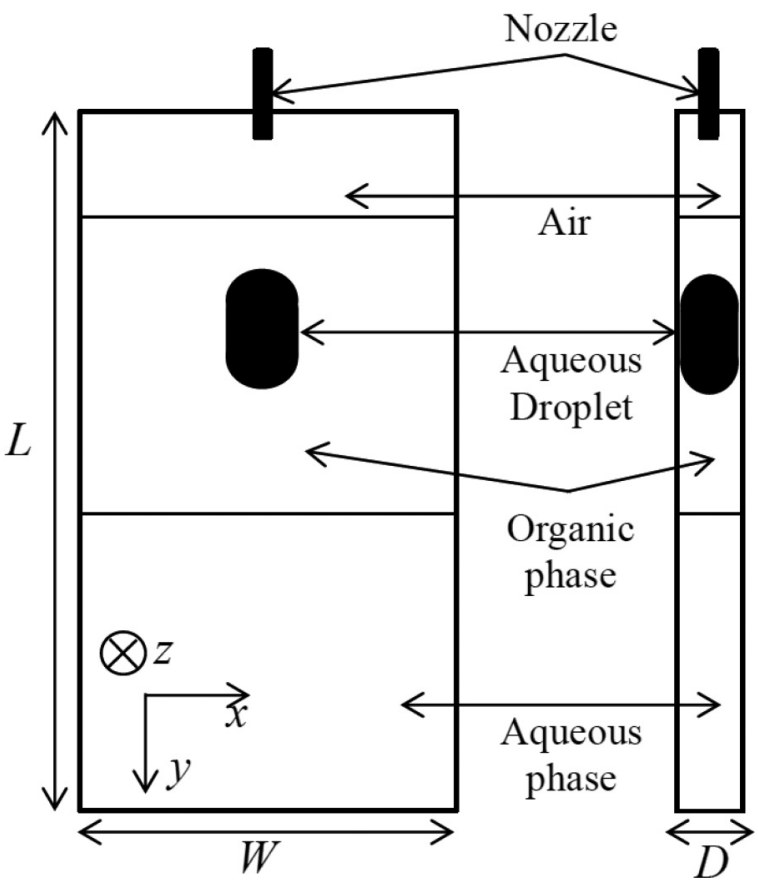

FIG. 1. Schematic representation of the experimental setup.

velocity component and the $x$ and $y$ components suggests that the flow in the $x-y$ plane can be considered as two dimensional.

In the cell, the coalescence of an aqueous 2D droplet with an aqueous-organic interface is studied. The aqueous phase is a $76 \% \mathrm{v} / \mathrm{v}$ glycerol-water mixture, while the organic phase is a low-viscosity, kerosenelike oil (Exxsol D80). The glycerol concentration in the aqueous phase solution was chosen to match the refractive index of the organic phase, necessary for optical based flow measurements [28]. Refractive indexes were measured with a refractometer (Abbe 5, BS) and were found to be $r_{\text {Exxsol }}=1.443\left(22^{\circ} \mathrm{C}\right)$ for Exxsol D80 and $r_{\text {glyc } / \text { wat }}=1.443\left(22^{\circ} \mathrm{C}\right)$ for the aqueous phase. By matching the refractive indexes of the two phases it was possible to improve the quality of the measurements very close to the interface, but at the same time it was difficult to trace the interface. A very small amount of ink was added in the aqueous phase to improve the contrast between the two phases and facilitate the tracking of the interface. By using ink, the interface appears on the images as a sharp line of 1 pixel thickness $(10 \mu \mathrm{m})$.

Different concentrations of Span 80 (density of $990 \mathrm{~kg} / \mathrm{m}^{3}$ at $20^{\circ} \mathrm{C}$ and a molar weight of $428.6 \mathrm{~g} / \mathrm{mol}$ ) were introduced in the organic phase to modify the interfacial tension of the system. Span 80 is a nonionic surface active agent with an $\mathrm{HLB}=4.3$ rendering it more soluble in the organic phase and practically insoluble in the aqueous phase. The surfactant solutions were prepared by diluting an initial dense solution to the appropriate level. The solutions were then put in an ultrasound bath for $15 \mathrm{~min}$ at $45 \mathrm{kHz}$ to achieve better mixing. The properties of the two phases are given in Table I. The interfacial tension values of the final solutions containing the ink were measured with the pendant drop method. For the validation of the interfacial tension measurements the equation of state derived from the Langmuir adsorption isotherm was
TABLE I. Fluid properties.

\begin{tabular}{lcccc}
\hline \hline & $\begin{array}{c}\mu \\
\mathrm{mPas}\end{array}$ & $\begin{array}{c}\rho \\
\mathrm{Kg} \mathrm{m}^{-3}\end{array}$ & $\begin{array}{c}\text { Span } 80 \\
\mathrm{w} / \mathrm{w}\end{array}$ & $\begin{array}{c}\Gamma \\
\mathrm{mN} \mathrm{m}^{-1}\end{array}$ \\
\hline $\begin{array}{l}\text { Glycerol/water } \\
\text { (76\% v/v) }\end{array}$ & \multirow{2}{*}{54} & \multirow{2}{*}{1210} & & \\
& & & 0 & 26.7 \\
& & & $2 \times 10^{-5}$ & 21.1 \\
Exxsol D80 & \multirow{2}{*}{1.75} & \multirow{2}{*}{804} & $1 \times 10^{-4}$ & 18.3 \\
& & & $1.5 \times 10^{-4}$ & 13.2 \\
& & & $2 \times 10^{-4}$ & 9.6 \\
\hline \hline
\end{tabular}

used, which is given by

$$
d \gamma=-R T \Gamma_{\infty}\left(\frac{K_{L} c}{1+K_{L} c}\right) d \ln c
$$

The values for the equilibrium constant $K_{L}$ and the adsorption capacity $\Gamma_{\infty}$ for Span 80 are taken equal to $K_{L}=10 \mathrm{~m}^{3} / \mathrm{mol}$ and $\Gamma_{\infty}=4.42 \times 10^{-6} \mathrm{~mol} / \mathrm{m}^{2}$, following the suggestion of Giribabu and Ghosh [17] for mineral oilwater systems. As can be seen in Fig. 2 the experimental data on interfacial tension for different Span 80 concentrations in oil agree well with the predictions of Eq. (1) with the parameter given by Giribabu and Ghosh [17]. Interfacial tension measurements showed that any mass transfer between the aqueous-ink and organic phase are negligible as $\gamma$ does not change in the time scale of $24 \mathrm{~h}$.

For the coalescence experiments, the aqueous phase was put into the cell up to approximately $4 \mathrm{~cm}$ from the bottom and the organic phase was added on top up to a final height of $8 \mathrm{~cm}$. The aqueous drops were generated with a syringe pump (KDS Scientific) and formed at a metallic nozzle of $0.5 \mathrm{~mm}$ ID. The addition of surfactant in the oil phase would change the size of the drops generated at the nozzle because it affects the interfacial tension between the two fluids and the contact angle with the nozzle [34]. To overcome this problem, drops were generated in air above the oil layer and were then introduced into the oil phase. It was found that the volume of the drops generated in the air was approximately constant for all surfactant concentrations used and equal to about $0.7 \mathrm{ml}$. For a constant oil layer thickness, the seeding time,

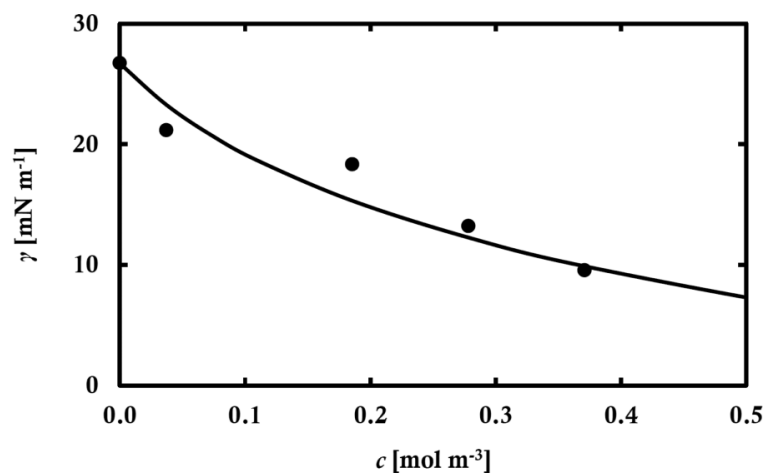

FIG. 2. Interfacial tension values of Exxsol D80 and a waterglycerol mixture for different Span 80 concentrations. The continuous line represents Eq. (1). 
which is the time until the drop reaches the organic-aqueous interface from the moment it enters the organic phase, was approximately equal to $30 \mathrm{~s}$. At high surfactant concentrations (above $2 \times 10^{-4} \mathrm{w} / \mathrm{w}$ ) the drops generated in the air broke into two smaller ones upon entering the oil layer because of a Plateau-Rayleigh instability. Surfactant concentrations above this one were therefore not used in this work to keep the drop volume constant. A small valve was placed at the bottom of the cell to regulate the static pressure and keep the organic-aqueous interface as flat as possible.

The droplet coalescence process with a flat interface in a Hele-Shaw cell was studied using high-speed video recording. The camera (Photron APX) was equipped with a monozoom Nikon lens giving a camera depth of field (DOF) approximately equal to $0.5 \mathrm{~mm}$ and a $3 \times$ magnification. The focal plane was placed in the center of the channel. Velocity fields during coalescence were acquired with a high-speed shadowgraphy system [32]. In this technique, the channel is illuminated with a uniform white backlight and images of the tracer particles added in the aqueous phase are recorded by the high-speed camera placed in front of it. Velocity fields are calculated by cross-correlating the movement of the particles between two successive frames of the high-speed camera. The measurements are based on the black and white (BW) signal contrast of the tracer particles. The tracers used are $10-\mu \mathrm{m}$ silver coated glass particles. This technique is equivalent to high-speed PIV suitable for small scale flows, but without the use of a laser. In high-speed PIV measurements in large scale flows, the plane of measurement is defined by the thickness of the illuminating laser sheet. In this case, the DOF of the camera is generally one order of magnitude larger than the laser sheet thickness. In small scale flows, however, the use of microscope lenses reduces significantly the DOF (values between 1 and $1000 \mu \mathrm{m}$ ), rendering it thinner than the laser sheet thickness. The plane of measurement is then defined by the DOF of the camera while a laser, or in this work a white light, provides volume illumination.

The movement of the particles along the $z$ direction and inside a PIV correlation box, due to shear, can produce erroneous results [35]. The stratification of the velocity in the depth of field of the camera is estimated at $25 \%$ of the maximum velocity, assuming a parabolic velocity profile along the $z$ direction, so the correlation errors are estimated to be very low. The images are treated within a commercial PIV software (INSIGHT 4G, TSI). The correlation grid size was fixed to $32 \times 32$ pixels with $50 \%$ spatial overlap which gives a final spatial resolution of about $0.16 \times 0.16 \mathrm{~mm}$, while the sampling frequency of the camera was kept constant at $2 \mathrm{kHz}(\Delta t=$ $0.5 \mathrm{~ms}$ ). A grid deformation code and a secondary-to-primary correlation peak ratio as threshold were used to exclude false vectors. A postprocessing treatment was applied to replace false vectors with the local median value. Data were analyzed with MATLAB codes developed in house.

\section{RESULTS AND DISCUSSION}

\section{A. Interfacial dynamics: Evolution of the neck}

From the high-speed images it was found that the presence of surface active agents modifies the geometry of the approach- ing interfaces compared to systems without surfactants. This is demonstrated in Fig. 3 where schematics of the geometrical parameters relevant to droplet coalescence with and without a surfactant present in the oil phase are shown, assuming a 2D configuration. Raw images for droplet coalescence without surfactant [Fig. 3(a)] and with a $1.5 \times 10^{-4} \mathrm{w} / \mathrm{w}$ surfactant concentration [Fig. 3(b)] are used to illustrate the corresponding configurations. Without a surfactant [Fig. 3(c)], the neck (radius $r$ ) connecting the drop with the interface is located above the initial flat interface level during the whole coalescence process. The distance $h$, separating the center of the neck from the initial interface height, corresponds to the local radius of curvature of the neck during the early stages of coalescence. A similar geometrical modeling has been used by Yokota and Okumura [23] to describe the dynamics of droplet coalescence for different viscosity fluids in a Hele-Shaw cell. When a surfactant is present in the bulk phase [Fig. 3(d)] the geometrical characteristics change. The addition of surfactant decreases the interfacial tension that keeps the interface flat which then tends to deform under the approaching drop. In this case, the horizontal plane defined by the neck radius, $r$, is located for a given time below the initial interface level. During the initial stages of neck rupture and until the neck reaches the initial interface height, the local radius of the neck curvature is not large enough to be detected by the current imaging system. In these stages the neck appears on the images as a curved line moving along the contact line between the drop and the lower phase.

To study the dynamics of coalescence, the evolution of the neck radius over time is presented in Fig. 4. The data follow a linear trend irrespective of the surfactant concentration. More importantly, it can be seen that this linearity continues for neck diameters beyond the depth of the cell, which is in accordance with previous data in Hele-Shaw cell geometries [22,23]. This linear evolution of the neck with time indicates an almost constant interfacial tension value at least at the neck region during the initial stage. This suggests that there is no variation of the surfactant concentration at the neck during this stage. From the slopes of the lines in Fig. 4 the neck expansion velocities, $V$, can be calculated for the different surfactant concentrations. It should be noted that in Fig. 4 the data of only one typical drop coalescence event are shown for each concentration, but to produce the final dynamics many drops were used to obtain an average neck velocity for each concentration. The average neck velocity for the pure solution is $295( \pm 45 \%) \mathrm{mm} / \mathrm{s}$. The deviation in the velocity is in fact much higher for the pure solutions compared to the surfactant ones (less than 10\%) for two main reasons. The pure solutions have higher neck velocities compared to the surfactant ones because they have higher interfacial tension which drives coalescence. With the image acquisition frequency used of $2 \mathrm{kHz}$ only five images could be captured for pure solutions in the first regime compared to approximately 30 for the highest concentrated surfactant solution. Secondly, the pure system is more susceptible to dust present in the cell compared to the surfactant ones (as also reported by [3]), which can affect the final neck expansion velocity. Similar velocity deviations have also been reported by Eri and Okumura [22], where for one set of liquids, neck velocities ranged from 250 to $350 \mathrm{~mm} / \mathrm{s}$.

Two distinct regimes which describe the neck expansion during droplet coalescence have been identified in previous 

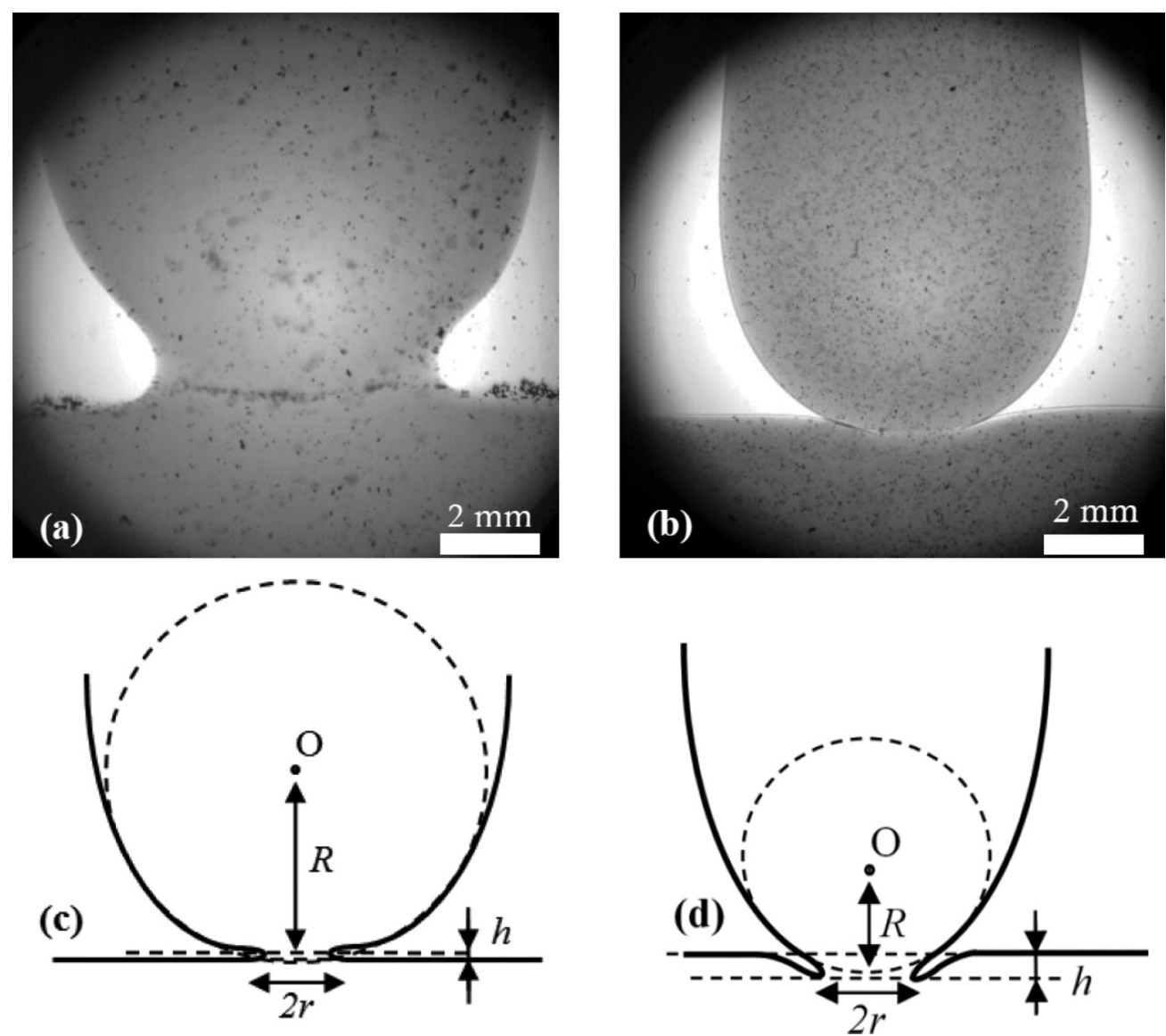

FIG. 3. Raw images of droplet coalescence (a) without surfactant and (b) for $1.5 \times 10^{-4} \mathrm{w} / \mathrm{w}$ surfactant concentration. Schematic of droplet coalescing on a flat interface (c) without surfactant and (d) with surfactant.

studies as discussed in Sec. I. The first, viscous, regime occurs immediately after the neck ruptures and is limited to a short time range. Even though coalescence takes place in a quasi-two-dimensional cell, to model the neck dynamics in this first regime, the flow is considered three dimensional, as the neck is smaller than the depth of the cell in the $z$ direction $[22,23]$. Following these assumptions, a general scaling law was developed which holds true for neck diameters $2 r \leqslant$ $D$ where $D$ represents the cell depth. The nondimensional

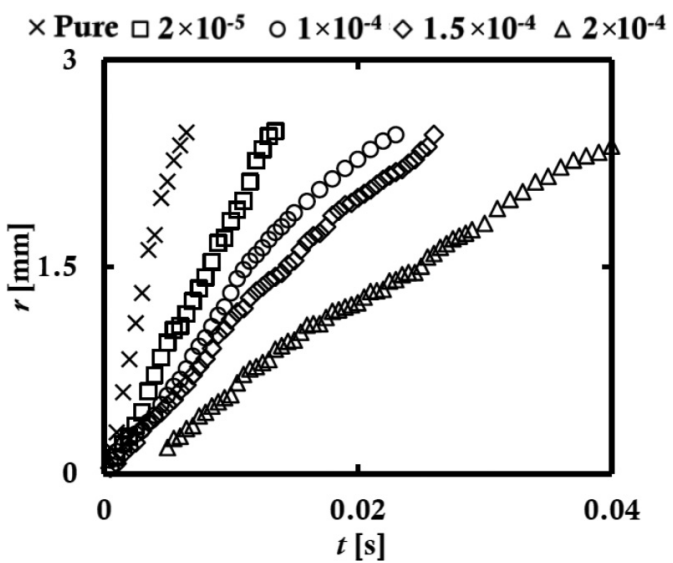

FIG. 4. Neck radius evolution over time of a typical drop for different surfactant concentrations (expressed in mass ratios). capillary, $\mathrm{Ca}$, and Ohnesorge, $\mathrm{Oh}$, numbers relevant in this regime are defined as follows:

$$
\begin{gathered}
\mathrm{Oh}=\frac{\mu}{\sqrt{R \rho \gamma}}, \\
\mathrm{Ca}=\frac{\mu V}{\gamma},
\end{gathered}
$$

where $\mu, \rho$, and $\gamma$ represent, respectively, the viscosity and the density of the aqueous phase and the interfacial tension between the aqueous and the organic phase. The characteristic velocity in the Ca number is the neck velocity $V=d r / d t$ while the characteristic length scale for the Oh number is the drop radius, which controls the surfactant diffusion dynamics.

A linear scaling law applies in the viscous regime (see Sec. I) where $\mathrm{Ca} \approx 1$, expressed as $r(t)=D\left(t / \tau_{i}\right)$ where $\tau_{i}=$ $D \mu / \gamma$; this gives $V=\gamma / \mu$ [23], which for the initial stages is valid for $3 \mathrm{D}$ as well as for $2 \mathrm{D}$ geometries for neck diameters less than the cell depth [12]. While this scaling law is well established for 3D liquid-liquid coalescence, it is not well explored for 2D cells. In 2D cells, a correction factor, $\alpha$, has been introduced in the law by Eri and Okumura [22] as follows:

$$
\begin{gathered}
V=\alpha \frac{\gamma}{\mu}, \\
\alpha=\frac{1}{2 \pi}\left[\ln \left(\frac{8}{\operatorname{Re}}\right)-c+0.5\right],
\end{gathered}
$$



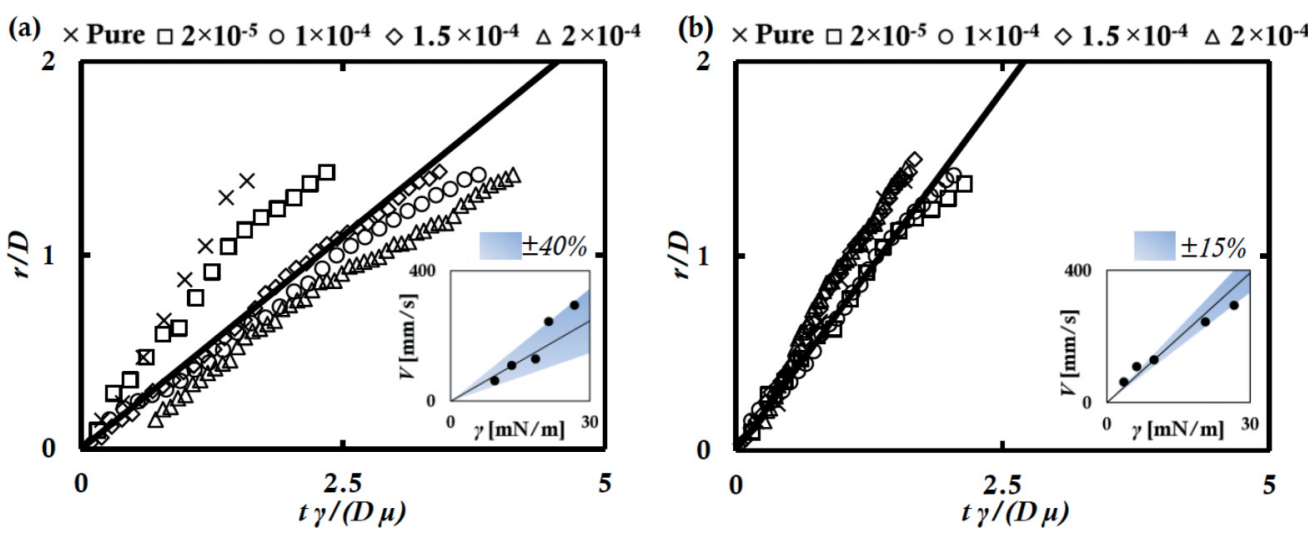

FIG. 5. (a) Scaled neck radius evolution over scaled time for (a) surfactant concentration at the neck equal to the bulk one, and (b) surfactant concentration at the neck double the bulk one. The insets show the corresponding averaged velocities for each concentration under each case. The continuous black lines in the inset represent the fitted slopes for (a) $\alpha=0.4$ and (b) $\alpha=0.7$, where $\alpha$ is the correction factor of the linear law.

where $\operatorname{Re}=2 \rho h^{\prime} V / \mu$ represents the Reynolds number, $h^{\prime}$ the local radius of curvature of the neck, and $c$ the Euler constant, equal to 0.577 . The correction factor $a$ corresponds to the drag coefficient of a cylinder in Stokes flow [36], and is found by considering that the neck is locally cylindrically shaped with a length equal to the cell depth and a radius equal to the radius of curvature of the neck. The parameter $a$ depends only on the local neck radius of curvature and the neck velocity, which can be considered constant for each surfactant concentration (as shown in Fig. 4). For the early stages of coalescence, the maximum local neck radius of curvature is about 2 pixels for all solutions, which in turn gives a value of approximately $h^{\prime}=20 \mu \mathrm{m}$. For the highest neck expansion velocity (pure solution) and the maximum radii of curvature, Eq. (5) gives for a system without surfactant a theoretical value of $\alpha=0.5$. The addition of surfactant can only decrease the neck expansion velocity and thus increase the theoretical value of $\alpha$.

Using the linear law, the nondimensional neck radius over the nondimensional time is plotted in Fig. 5(a). For the calculations the surfactant concentration at the neck region is taken equal to the bulk one (i.e., Table I). As shown in Fig. 5(a) the lines for the surfactant concentrated solutions clearly fail to collapse. The average velocities are also plotted against $\gamma$ in the inset of Fig. 5(a) where it can be seen that the slope of the line [correction factor $\alpha$ in Eq. (4)] is equal to 0.4 with a wide spread of data (approximately 40\%). This value is lower than the theoretical value for the pure solution $(\alpha=0.5)$ and cannot be correct as an increased surfactant concentration can only decrease the neck velocity and thus only increase $\alpha$.

Martin and Blanchette [21] showed with numerical simulations of a 3D droplet coalescing on a liquid-liquid interface that the surfactant concentration at the neck region during the early stages of coalescence is higher than the one in the bulk. As also described by Eggleton and Stebe [37], certain physical processes controlling the diffusion and desorption or adsorption of the surfactants have to be considered to better explain their effect on the dynamics of coalescence. In the case of a droplet coalescing with a flat interface, mass transfer of the surfactants both along the interface and from the bulk to the interface need to be taken into account. Dilution of the surfactant due to the interface dilatation can be ignored since there is no growth of interface during coalescence. In addition, the mass transfer of surfactant from the bulk to the interface is negligible for low surfactant concentrations [below Critical Micelle Concentration (CMC) value]. This is because mass transfer occurs mainly during the seeding time of the droplet, which is of the order of magnitude of $100 \mathrm{~s}$, compared to the coalescence process which is of the order of $1 \mathrm{~s}$ [21].

The current system is characterized by Ohnesorge numbers of the order of $10^{-1}$ which give $\mathrm{Pe}_{\Gamma}^{-1} \ll \mathrm{Oh}$, where $\mathrm{Pe}_{\Gamma}=$ $R^{2} /\left(D_{\Gamma} T\right)$ represents the Péclet number dependent on the surfactant concentration, while $D_{\Gamma}$ and $T$ are, respectively, the surface diffusivity and the typical time scale of diffusion [37]. In similar conditions, Martin and Blanchette [21] showed that for inert surfactant molecules, a peak of surfactant concentration appears at the neck during the coalescence process. They attributed this to the significant time difference between the typical time of surfactant diffusion and the time scale of the coalescence process. An interfacial tension gradient was computed along the interface away from the neck but locally the interfacial tension was constant.

According to the simulations by Martin and Blanchette [21], a concentration of surfactant almost double the bulk is expected during coalescence for the experiments presented here. From Fig. 2, the local interfacial tension values can be found at the neck, by doubling each bulk surfactant concentration and thus a new scaling curve can be produced, which is presented in Fig. 5(b). All curves now collapse in a single line, which suggests that locally at the neck the surfactant concentration is higher and approximately double that in the bulk solution. Additionally, the new fitted slope of $V$ versus $\gamma$ from the average neck velocity data [inset in Fig. 5(b)] gives a value of $\alpha$ equal to 0.7 , with small deviation $( \pm 15 \%)$. This value is above the theoretical one for the pure solution $(\alpha=0.5)$ and captures the slower neck evolution with surfactants.

This approach, however, of fitting the surfactant concentrated solutions to a linear law does not take into account the elasticity of the interface. Because of this elasticity, a liquid film is trapped between the top of the droplet and the interface. It is worth noting that for all the cases shown before this liquid film is relatively short and does not affect the neck dynamics. At high concentrations though, the film trapped 

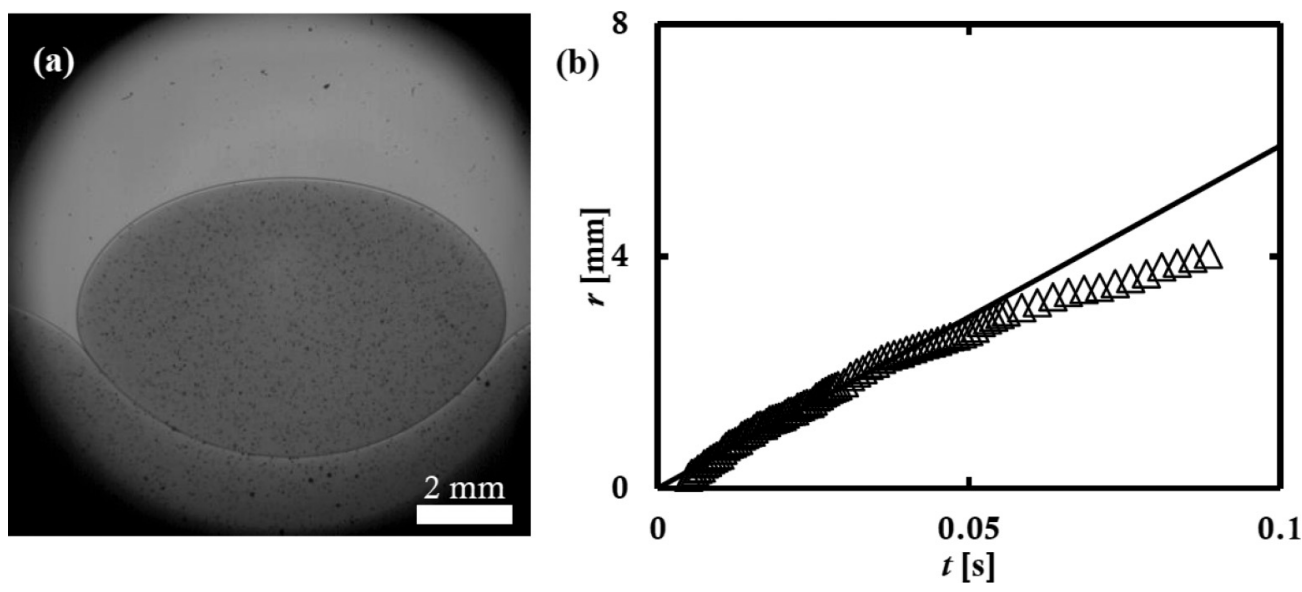

FIG. 6. (a) Image of an aqueous droplet surrounded by an organic phase with $2 \times 10^{-4}$ (w/w) Span 80 . (b) Neck evolution of this solution over the time.

is long and its presence cannot be neglected. Figure 6(a) illustrates the coalescence of a drop surrounded by an organic phase containing $2 \times 10^{-4} \mathrm{w} / \mathrm{w}$ of Span 80 . At this surfactant concentration the drop penetrates the bulk aqueous phase significantly before coalescence and the interface level reaches approximately the middle of the droplet. This behavior can be attributed to the liquid film that is trapped between the droplet and the bulk aqueous phase. This interface distortion can potentially result in a considerable movement of the neck in the $y$ direction. However, for short times after the film rupture, the motion of the neck is mainly directed along the $x$ axis. The motion along the horizontal $x$ direction was used to calculate the neck velocities. Figure 6(b) presents the time evolution of the neck radius during the coalescence for longer times to show the limit of the above approximation where the line represents a fitted linear evolution of the neck.

\section{B. Generation and advection of vortices}

In this section of the Results, all results presented correspond to a typical drop for each surfactant concentration. Streamlines are computed from the velocity fields obtained by

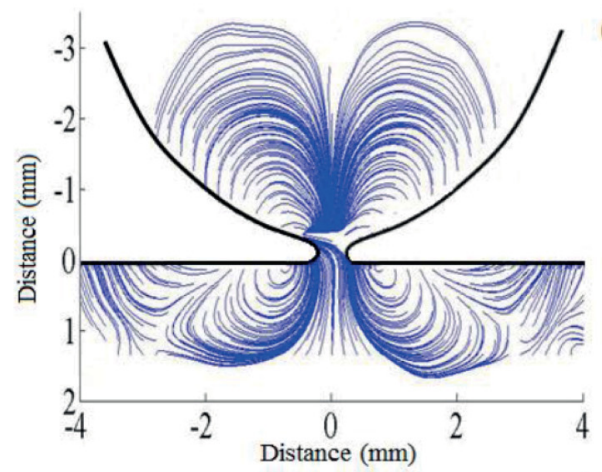

(a)

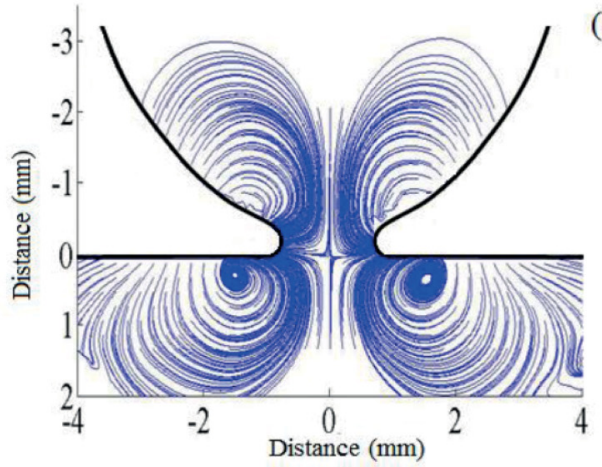

(b)

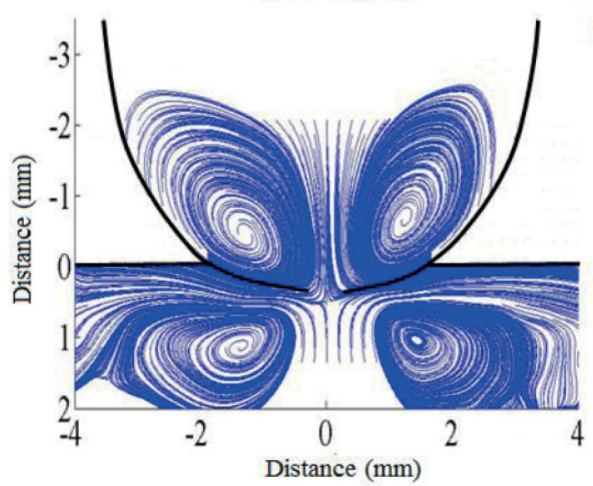

(c)

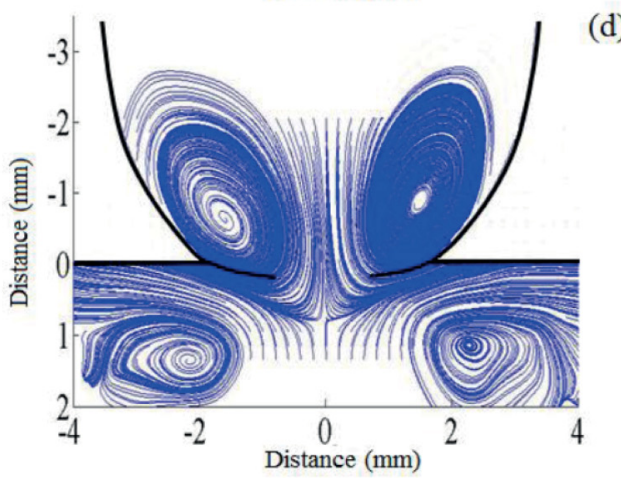

(d)

FIG. 7. Streamlines for different time steps corresponding to neck diameters equal to (a,c) $0.5 \mathrm{~mm}$ and (b,d) $1.25 \mathrm{~mm}$. The first set of images $(\mathrm{a}, \mathrm{b})$ corresponds to a droplet coalescing without surfactant while the second $(\mathrm{c}, \mathrm{d})$ corresponds to a droplet coalescing in a surfactant solution of $1.5 \times 10^{-4} \mathrm{w} / \mathrm{w}$. The interface has been plotted manually from the raw images to illustrate the approximate drop boundaries. 
(a)

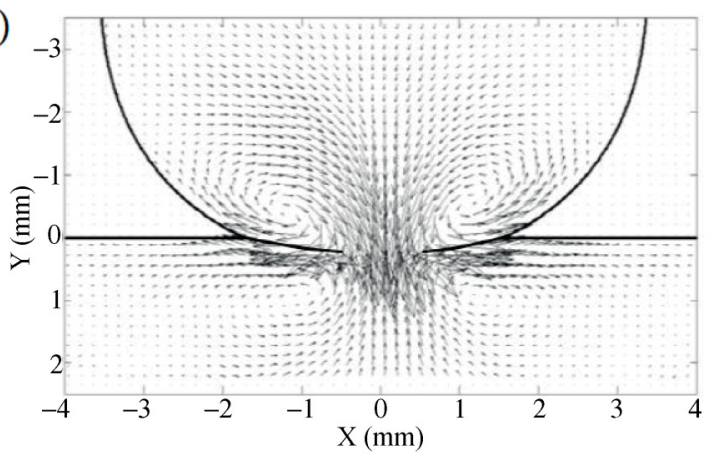

(c)

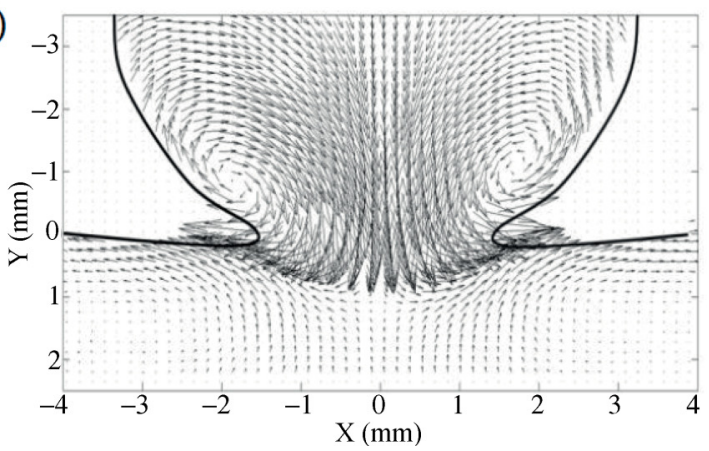

(b)

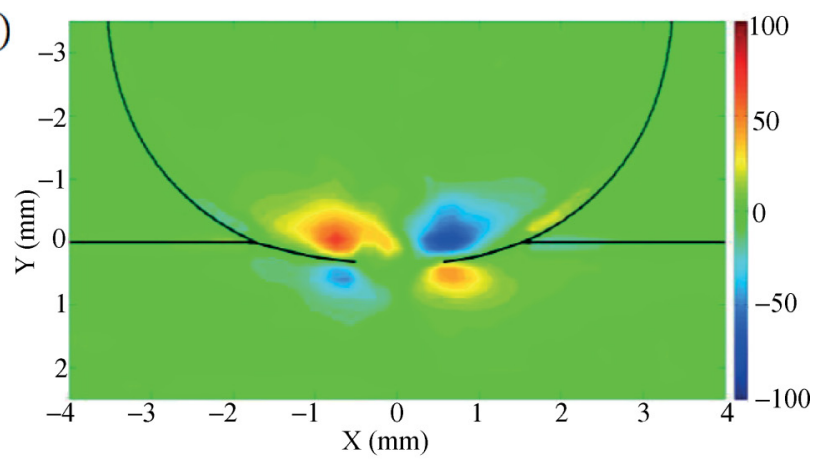

(d)

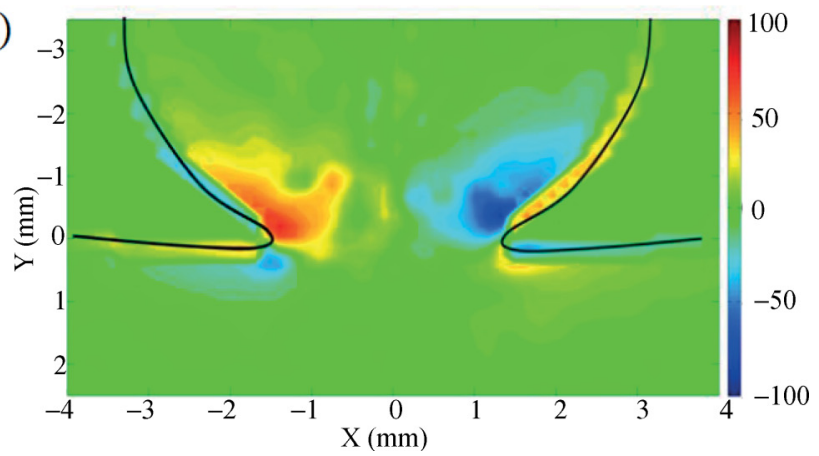

FIG. 8. Velocity field in the drop and bulk phase for a surfactant solution of $1.5 \times 10^{-4} \mathrm{w} / \mathrm{w}$ for (a) $2 r / D=0.8$, (c) $2 r / D=2$, and the corresponding vorticity isocontours for (b) $2 r / D=0.8$, (d) $2 r / D=2$. The interface has been plotted manually from the raw images to illustrate the approximate drop boundaries.

the PIV measurements with time steps of $0.5 \mathrm{~ms}$. For all the solutions implemented in this study, the hydrodynamics tends to exhibit the same pattern, despite the changes in the surfactant concentration in the organic phase. The moment the interface breaks and the coalescence singularity occurs, movement of the particles close to the singularity can be observed. The measurement of the coalescence time starts from this point. However, at the very early stages of coalescence, the bursting tip appears as a continuous black line and velocity fields and the neck radius cannot be computed. This behavior is more pronounced at high surfactant concentrations and corresponds to a maximum of two frames. Consequently the first velocity field measurements are taken after $0.5-1 \mathrm{~ms}$ depending on the surfactant concentration.

Two time steps corresponding to the viscous regime are shown in Fig. 7, where the effects of time and surfactant concentration can be observed. The time steps chosen correspond to neck diameters of $2 r=0.5$ and $1.25 \mathrm{~mm}$ or aspect ratios of $2 r / D=0.4$ and $2 r / D=1$, respectively. The velocity fields show the generation of two pairs of counter-rotating vortices. One pair is located inside the droplet above the initial interface level, while the other is formed in the bulk. The upper pair of vortices is notated as UV and the lower pair as LV, with the clockwise rotation as "-" and the anticlockwise as "+". As the time increases, UV- and UV+ are advected along the interface and to the top of the droplet, while LV+ and LV-are advected towards the bulk but stay close to the interface.

Qualitatively, the size of the vortices seems to increase in the surfactant concentrated solution. By continuity of the velocity, this can be attributed to the fact that a larger part of the interface is moving. The velocities of advection of the pairs
UV and LV are different and this behavior can be observed, for example, in the asymmetry between UV- and LV+ from the initial horizontal interface level which increases over time [Fig. 7(d)]. UV generation can be explained by considering that the expansion of the neck introduces two main flow velocities inside the droplet-one horizontal in the direction of the neck growth, and the other vertical with direction from the droplet to the bulk. These two components generate, respectively, $\mathrm{UV}+$ and UV-. For the generation of LV+ and LV- a similar reasoning can be applied. From the singularity point where the rupture occurs, two vertical flow movements (positive for UV and negative for LV) are generated. This results in significant pressure difference between this singularity point and the rest of the fluid, which drives the neck growth and the vertical velocity components.

From the PIV measurements conducted in this study, it is shown that the upward and downward vertical velocity components, both directed towards the singularity point, are of the same order of magnitude for very short times. Interestingly though, for longer times, the downward velocity becomes higher than the upward as the coalescence progresses. Previous experimental efforts on the coalescence of 3D droplets [28] have not managed to capture these short time dynamics but have illustrated a similar behavior for the longer times.

For a given solution, LV and UV have different advection velocities which depend on the surfactant concentration. To quantify the velocity difference, vortices have been tracked. Figure 8 (a) presents a typical velocity field for a surfactant concentration of $1.5 \times 10^{-4} \mathrm{w} / \mathrm{w}$ while Fig. $8(\mathrm{~b})$ shows the vorticity isocontours. It can be noticed that there is a slight deviation between the centers of the vortices and the location 


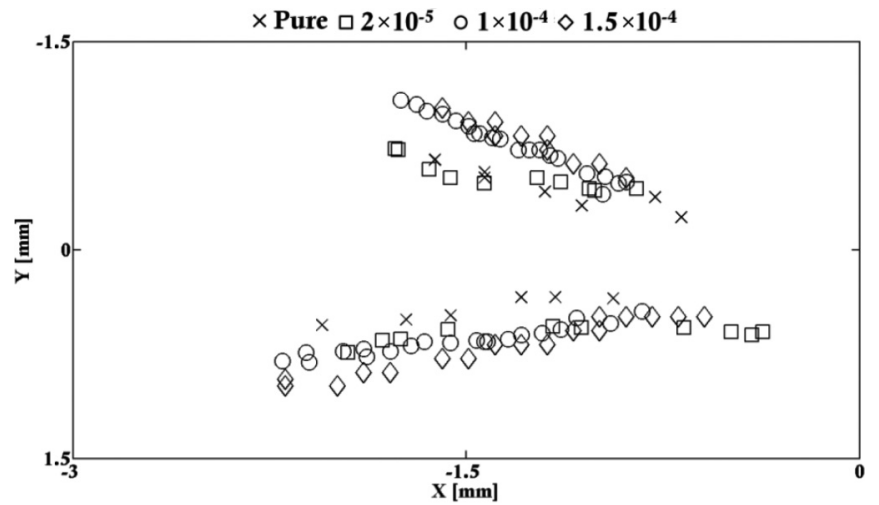

FIG. 9. Spatial evolution in the $x-y$ plane of the centers of the two vortices present on the left-hand side of the droplet (UV-for $y<0$ and $\mathrm{LV}+$ for $y>0$ ). The different symbols represent the time evolution of the position of the centers of the two vortices for a constant time step within the viscous regime. Results are shown for different surfactant concentrations, $1.5 \times 10^{-4}(\diamond), 1 \times 10^{-4}(\circ), 2 \times 10^{-5}(\square)$, and pure solution $(\times)$ expressed in mass ratios.

of the vorticity extrema. Figures 8 (a) and 8(b) exhibit clearly that the vorticity isocontours mainly match the internal "corner" of the vortex position which corresponds to the rotation occurring close to the singularity. It is worth noting that $\mathrm{LV}+$ and $\mathrm{LV}$ - vanish as the end of the viscous regime is reached, as is shown in Figs. 8(c) and 8(d).

The tracking of the vortices depends on the complexity of the flow (3D droplet coalescence, coalescence at a moving interface, etc.) [38] and in the present study the streamlines seem to indicate better the location of the centers of the vortices, rather than other computational methods. The centers of the vortices have been tracked manually at each time step by recording the position of the center of the core created by the streamlines with an accuracy of a corresponding PIV correlation box $( \pm 0.16 \mathrm{~mm})$. Figure 9 presents for one drop the spatial evolution in the $x-y$ plane, of the centers of the two vortices on the left-hand side (UV- and LV+) for a constant time step within the viscous regime. The upper part of the graph (negative $y$ axis) corresponds to the movement of UVwhile the lower part of the graph (positive $y$ axis) corresponds to the movement of $\mathrm{LV}+$. The data have been rescaled by putting the origin of the frame in the position of the initial interface breaking point. This is because the breakage point does not always occur at the center of the drop.

The presence of the surfactant tends to shift the trajectory of the UV vortices to the top of the droplet over time. As shown in Fig. 9, UV- tends to have a linear trend for all cases while the slope is increased at higher surfactant concentrations. The distance traveled over time for one drop of each concentration can be seen in Fig. 10 for the UV- [Fig. 10(a)] and LV+ [Fig. 10(b)] vortices. The propagation seems to be linear, which indicates that the velocity of each vortex remains constant. For each solution, the velocity of advection of $\mathrm{LV}+$ is always greater than the velocity of advection of UV-. This can be qualitatively explained by considering that UV is bounded by the aqueous-organic interface with a shape that depends significantly on the surfactant concentration. It can also be seen that for one drop the advection velocities of UV- are
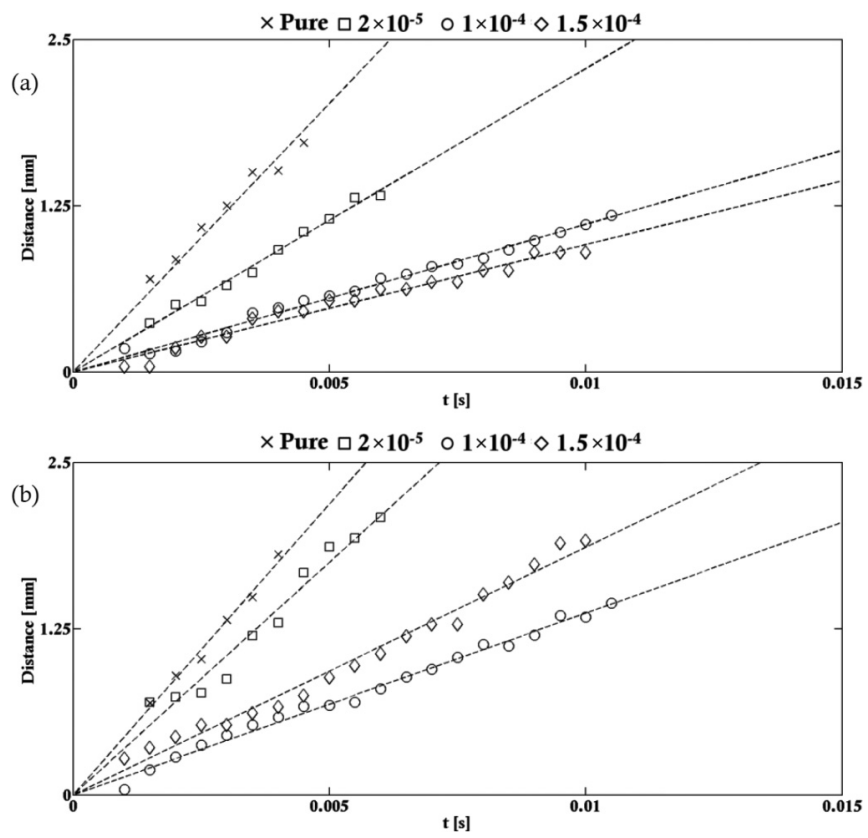

FIG. 10. (a) UV- distance traveled versus time. (b) $\mathrm{LV}+$ distance traveled versus time. Results are shown for different surfactant concentrations, $1.5 \times 10^{-4}(\diamond), 1 \times 10^{-4}(\circ), 2 \times 10^{-5}(\square)$, and pure solution $(\times)$ expressed in mass ratios.

slightly lower than the corresponding neck velocities, while those of LV+ are higher; this was observed for all drops studied. The neck velocity appears to be the mean of the two countervortices UV- and LV+ for each coalescing drop. The vortices generated by the rupture of the interface seem to follow the neck dynamics for the viscous regime. The trajectories of the vortices plotted are only shown for time up to $0.01 \mathrm{~s}$, compared to Fig. 4 , because at longer times the vortices collapse and the corresponding streamlines cannot be traced accurately.

The patterns illustrated in Fig. 7 indicate that the neck bridge region is submitted to high shear rates mainly due to the vortices present in that region. The computed shear rates $\partial u_{y} / \partial x$ are plotted in Fig. 11 for two neck widths and for two solutions-one without surfactants and the other with $1.5 \times$ $10^{-4} \mathrm{w} / \mathrm{w}$ surfactant. As the flow is mainly driven along the $y$ axis it is interesting to investigate the shear of the $y$-velocity component along the $x$ axis. The magnitude of the shear seems to be slightly affected by the surfactant, but more importantly, there is an observable change in the location of the maxima. For the flow located above the neck region (i.e., inside the aqueous droplet), the cores surrounding the shear extrema are spatially smaller without surfactant compared to the Span 80 concentrated solution. The presence of the surfactant does not seem to modify significantly the mixing generated by the axial shear flow inside the droplet.

However, there is a significant difference of the shear pattern in the bulk phase when surfactant is added [Figs. 11(a) and 11(b)]. While the two main shear cores in the bulk are located in both cases around the neck, for the pure solution two secondary cores are also present close to the interface but far from the neck. These secondary shear peaks are due to a quick variation of the $y$ velocity along a short 
(a)

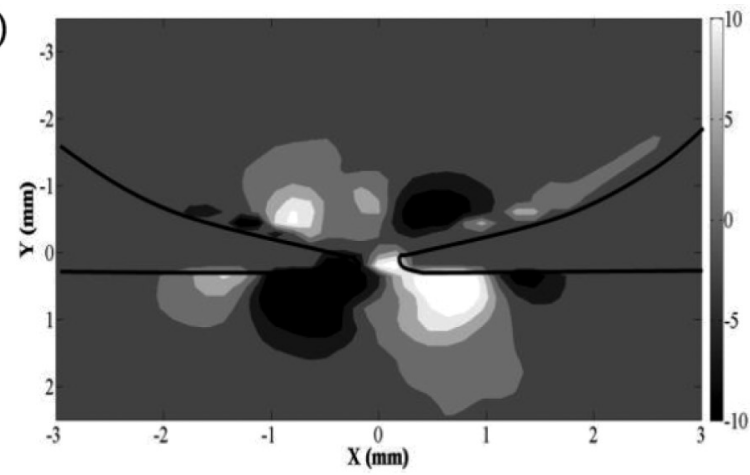

(b)

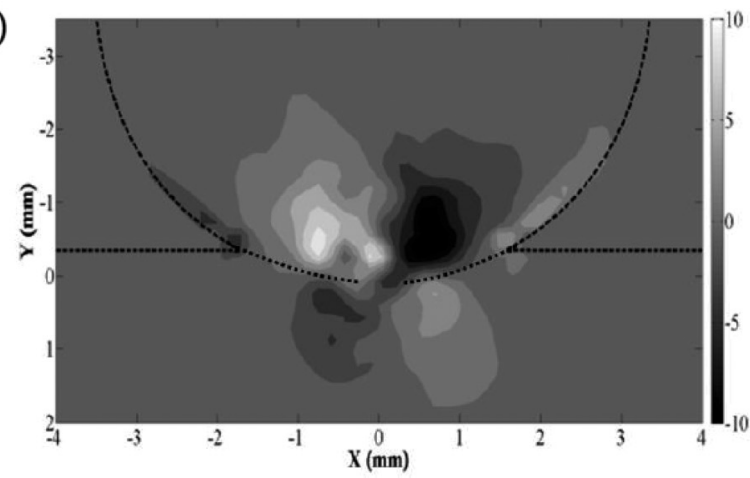

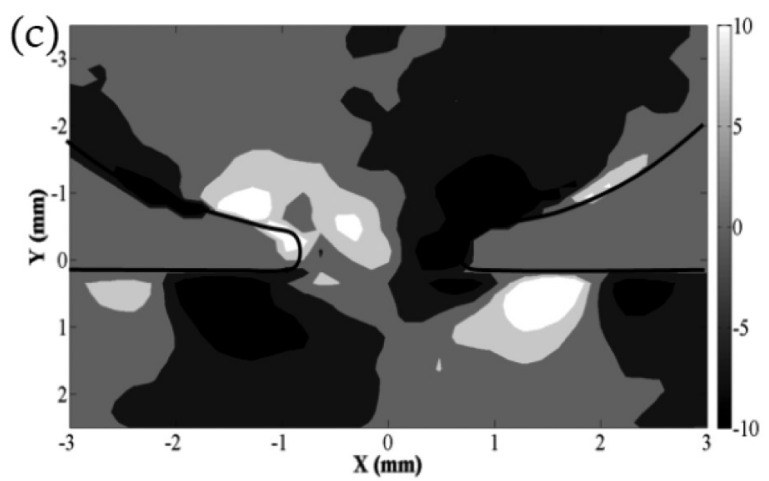

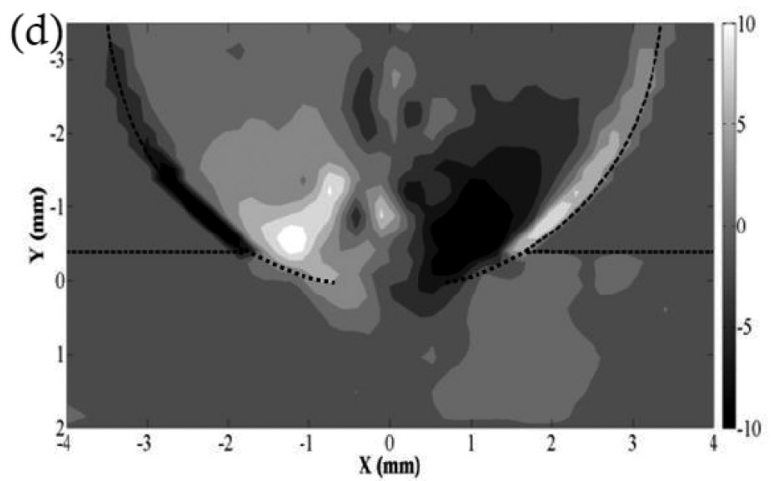

FIG. 11. (a) Shear rate $\partial u_{y} / \partial x$ evolution for the pure solution and a neck diameter equal to $0.5 \mathrm{~mm}$ and (c) $1.25 \mathrm{~mm}$. (b) A similar plot is shown for a surfactant solution $1.5 \times 10^{-4}(\mathrm{w} / \mathrm{w})$ for a neck distance equal to $0.5 \mathrm{~mm}$ and (d) $1.25 \mathrm{~mm}$. The interface has been plotted manually from the raw images to illustrate the approximate drop boundaries.

distance in the $x$ axis, generated mainly by the LV vortices. As illustrated previously, the addition of surfactant not only slows down the neck dynamics, but also modifies the elasticity of the interface, changing significantly the properties of the two vortex pairs LV and UV. The addition of the surfactant increases significantly the size of the vortices and decreases the shear $\partial u_{y} / \partial x$ inside the bulk. The mixing inside the bulk generated by the coalescence itself is decreased when the surfactant concentration is increased. Figures 11(c) and 11(d) present the shear generated when the neck diameter has grown to $1.25 \mathrm{~mm}$. The shear structures are then advected following the neck movement. In the pure case, the shear core structures present in the bulk are advected while they keep their amplitude. In the case of the surfactant solution, however, they tend to disappear. If the LV vortices are still present at this stage, their spreading along the $x$ axis is too large to be observed by the computation of the shear.

\section{SUMMARY AND CONCLUSIONS}

In this work, the coalescence of an aqueous drop with a liquid-liquid flat interface was investigated in a Hele-Shaw cell for different concentrations of an oil soluble nonionic surfactant. High-speed imaging revealed that the neck forming after the rupture of the film trapped between the drop and the interface expands linearly over time at the early stages of coalescence. However, the scaling law proposed by [22] for systems with no surfactants present does not agree with the current results. It is suggested, in agreement with very recent simulations [21], that the local surfactant concentration at the neck region has to be properly considered. An approximately double concentration of the surfactant is assumed locally at the neck, changing the interfacial tension values which have to be considered for the linear scaling of the velocity at the early stages of coalescence.

As previously shown in the literature [28], the 3D coalescence process generates vortices mainly in the bulk phase. In this work it was found that another pair of vortices is generated into the droplet and the velocity of advection of the vortices on each side of the droplet is similar to the neck velocity. The computed streamlines indicate that the vortices are generated by local reduction in pressure at the singularity point where the film ruptures. The presence of surfactants tends to increase the size of the vortices, but decreases their magnitude. The two opposing vertical velocity components towards the singularity point are of the same order of magnitude for short times. These opposing vortices generate very high shear rates at the region of the neck. The surfactant changes the magnitude of the velocity components but not that of the shear rates.

These results show the effect of surface active agents on the hydrodynamics of coalescence in the presence of surfactants. The linearity of the neck expansion for short times and surfactant concentrations lower than the CMC value will contribute to the understanding of the coalescence process in the presence of surfactants. In addition, the detailed measurements of the flow characteristics presented here can be used to develop and validate numerical codes of coalescence.

\section{ACKNOWLEDGMENTS}

This project was funded under the UK Engineering and Physical Sciences Research Council (EPSRC) 
Programme Grant MEMPHIS (EP/K003976). The authors would like to acknowledge the EPSRC Instrument Pool for the loan of the high-speed camera.
V.V. is grateful to Chevron Energy Technology Company and to University College London for their support in the form of a studentship.
[1] S. G. Huisman, P. Ern, and V. Roig, Phys. Rev. E 85, 027302 (2012).

[2] J. D. Paulsen, R. Carmigniani, A. Kannan, J. C. Burton, and S. R. Nagel, Nat. Commun. 5, 3182 (2014).

[3] E. d. Malmazet, F. Risso, O. Masbernat, and V. Pauchard, Colloids Surf. A 482, 514 (2015).

[4] H. P. Kavehpour, Annu. Rev. Fluid Mech. 47, 245 (2015).

[5] J. Lu, S. Fang, and C. M. Corvalan, Phys. Rev. E 93, 023111 (2016).

[6] J. Eggers, J. R. Lister, and H. A. Stone, J. Fluid Mech. 401, 293 (1999).

[7] J. E. Sprittles and Y. D. Shikhmurzaev, Phys. Fluids 24, 122105 (2012).

[8] A. Menchaca-Rocha, A. Martinez-Davalos, R. Nunez, S. Popinet, and S. Zaleski, Phys. Rev. E 63, 046309 (2001).

[9] S. T. Thoroddsen, B. Qian, T. G. Etoh, and K. Takehara, Phys. Fluids 19, 072110 (2007).

[10] J. D. Paulsen, J. C. Burton, and S. R. Nagel, Phys. Rev. Lett. 106, 114501 (2011).

[11] D. G. A. L. Aarts, H. N. W. Lekkerkerker, H. Guo, G. H. Wegdam, and D. Bonn, Phys. Rev. Lett. 95, 164503 (2005).

[12] J. C. Burton and P. Taborek, Phys. Rev. Lett. 98, 224502 (2007).

[13] M. M. Wu, T. Cubaud, and C. M. Ho, Phys. Fluids 16, L51 (2004).

[14] S. T. Thoroddsen, K. Takehara, and T. G. Etoh, J. Fluid Mech. 527, 85 (2005).

[15] L. Duchemin, J. Eggers, and C. Josserand, J. Fluid Mech. 487, 167 (2003).

[16] Y. Chen, C. Shen, and G. P. Peterson, Ind. Eng. Chem. Res. 54, 9257 (2015).

[17] K. Giribabu and P. Ghosh, Chem. Eng. Sci. 62, 3057 (2007).

[18] B. Dai and L. G. Leal, Phys. Fluids 20, 040802 (2008).
[19] L. Y. Yeo, O. K. Matar, E. S. P. de Ortiz, and G. E. Hewitt, J. Colloid Interface Sci. 257, 93 (2003).

[20] F. Blanchette, L. Messio, and J. W. M. Bush, Phys. Fluids 21, 072107 (2009).

[21] D. W. Martin and F. Blanchette, Phys. Fluids 27, 012103 (2015).

[22] A. Eri and K. Okumura, Phys. Rev. E 82, 030601(R) (2010).

[23] M. Yokota and K. Okumura, Proc. Natl. Acad. Sci. USA 108, 6395 (2011).

[24] P. G. Saffman and G. Taylor, Proc. R. Soc. London, Ser. A 245, 312 (1958).

[25] A. Eri and K. Okumura, Soft Matter 7, 5648 (2011).

[26] K. Fezzaa and Y. Wang, Phys. Rev. Lett. 100, 104501 (2008).

[27] S. C. Case and S. R. Nagel, Phys. Rev. Lett. 100, 084503 (2008).

[28] Z. Mohamed-Kassim and E. K. Longmire, Phys. Fluids 16, 2170 (2004).

[29] J. Kim and E. K. Longmire, Exp. Fluids 47, 263 (2009).

[30] A. D. Bordoloi and E. K. Longmire, Phys. Fluids 24, 062106 (2012).

[31] C. Ortiz-Duenas, J. Kim, and E. K. Longmire, Exp. Fluids 49, 111 (2010).

[32] M. Chinaud, E. P. Roumpea, and P. Angeli, Exp. Therm. Fluid Sci. 69, 99 (2015).

[33] M. Chinaud, T. Delaunay, S. Cazin, E. Cid, and P. Tordjeman, J. Non-Newtonian Fluid Mech. 169-170, 114 (2012).

[34] W. Wang, K. H. Ngan, J. Gong, and P. Angeli, Colloids Surf. A 334, 197 (2009).

[35] R. J. Adrian and J. Westerweel, Particle Image Velocimetry (Cambridge University Press, Cambridge, 2011).

[36] H. Lamb, Hydrodynamics (Cambridge University Press, Cambridge, 1932).

[37] C. D. Eggleton and K. J. Stebe, J. Colloids Interface Sci. 208, 68 (1998).

[38] J. Jeong and F. Hussain, J. Fluid Mech. 285, 69 (1995). 